\title{
A GENERALIZATION OF JUNG'S THEOREM
}

\author{
M. HENK
}

\begin{abstract}
The theorem of Jung establishes a relation between circumradius and diameter of a convex body. The half of the diameter can be interpreted as the maximum of circumradii of all 1-dimensional sections or 1-dimensional orthogonal projections of a convex body. This point of view leads to two series of $j$-dimensional circumradii, defined via sections or projections. In this paper we study some relations between these circumradii and by this we find a natural generalization of Jung's theorem.
\end{abstract}

\section{Introduction}

Throughout this paper $E^{d}$ denotes the $d$-dimensional euclidean space and the set of all convex bodies $K \subset E^{d}$ - compact convex sets - is denoted by $\mathcal{K}^{d}$. The affine (convex) hull of a subset $P \subset E^{d}$ is denoted by aff $(P)(\operatorname{conv}(P))$ and $\operatorname{dim}(P)$ denotes the dimension of the affine hull of $P$. The interior of $P$ is denoted by $\operatorname{int}(P)$ and $\operatorname{relint}(P)$ denotes the interior with respect to the affine hull of $P$. $\|\cdot\|$ denotes the euclidean norm and the set of all $i$-dimensional linear subspaces of $E^{d}$ is denoted by $\mathcal{L}_{i}^{d}$. $L^{\perp}$ denotes for $L \in \mathcal{L}_{i}^{d}$ the orthogonal complement and for $K \in \mathcal{K}^{d}, L \in \mathcal{L}_{i}^{d}$ the orthogonal projection of $K$ onto $L$ is denoted by $K \mid L$.

The diameter, circumradius and inradius of a convex body $K \in \mathcal{K}^{d}$ is denoted by $D(K), R(K)$ and $r(K)$, respectively. For a detailed description of these functionals we refer to the book $[\mathrm{BF}]$. With this notation we can define the following $i$-dimensional circumradii

Definition. For $K \in \mathcal{K}^{d}$ and $1 \leq i \leq d$ let

$$
\begin{aligned}
\text { i) } R_{\sigma}^{i}(K) & :=\max _{L \in \mathcal{L}_{i}^{d}} \max _{x \in L^{\perp}} R(K \cap(x+L)), \\
\text { ii) } R_{\pi}^{i}(K) & :=\max _{L \in \mathcal{L}_{i}^{d}} R(K \mid L) .
\end{aligned}
$$

We obviously have $R_{\sigma}^{i+1}(K) \geq R_{\sigma}^{i}(K), R_{\pi}^{i+1}(K) \geq R_{\pi}^{i}(K), R_{\pi}^{i}(K) \geq R_{\sigma}^{i}(K)$ and $R_{\sigma}^{d}(K)=R_{\pi}^{d}(K)=R(K), R_{\sigma}^{1}(K)=R_{\pi}^{1}(K)=D(K) / 2$.

The theorem of JUNG $[\mathrm{J}]$ states a relation between the circumradius and the diameter of a convex body. On account of the definition of $R_{\sigma}^{d}(K), R_{\sigma}^{1}(K)$ we can describe his result as follows

\footnotetext{
1991 Mathematics Subject Classification. AMS 52A43.

Key words and phrases. Circumradius, Diameter, Inradius.

I would like to thank Prof. Dr. J. M. Wills, who called my attention to these generalized circumradii
} 
Theorem of Jung. Let $K \in \mathcal{K}^{d}$. Then

$$
R_{\sigma}^{d}(K) \leq \sqrt{\frac{2 d}{d+1}} R_{\sigma}^{1}(K)
$$

and equality holds if and only if $K$ contains a regular $d$-simplex with edge length $D(K)$.

In the same way the theorem may be described with the circumradii $R_{\rho}^{d}(K)$ and $R_{\rho}^{1}(K)$. Here we study in general the relations between the $i$-dimensional and $j$-dimensional circumradius of these both series and get the following results

\section{Results}

Theorem 1. Let $K \in \mathcal{K}^{d}$ and $1 \leq j \leq i \leq d$. Then

$$
R_{\sigma}^{i}(K) \leq \sqrt{\frac{i(j+1)}{j(i+1)}} R_{\sigma}^{j}(K)
$$

and equality holds for $i>j$ if and only if $K$ contains a regular $i$-simplex with edge length $R_{\sigma}^{j}(K) \sqrt{\frac{2(j+1)}{j}}$.

Theorem 2. Let $K \in \mathcal{K}^{d}$ and $1 \leq j \leq i \leq d$. Then

$$
R_{\pi}^{i}(K) \leq \sqrt{\frac{i(j+1)}{j(i+1)}} R_{\pi}^{j}(K),
$$

and equality holds for $i>j$ if and only if an orthogonal projection of $K$ onto an $i$ dimensional linear subspace contains a regular $i$-simplex with edge length $R_{\pi}^{j}(K) \sqrt{\frac{2(j+1)}{j}}$.

Let us remark that both theorems are a generalization of the classical theorem of Jung since for $i=d, j=1$ the inequalities (1.2) and (1.3) become (1.1).

\section{Proofs}

To prove these theorems it is necessary to examine in more detail the circumradii of simplices since the circumradius of a convex body $K$ is determined by the circumradius of a certain simplex $\bar{T} \subset K$. This well known fact is described in the following lemma

Lemma 1. Let $K \subset \mathcal{K}^{d}$ and 0 be the center of the circumball of $K$. Then there exists a $k$-simplex $\bar{T} \subset K, \bar{T}=\operatorname{conv}\left(\left\{x^{0}, \ldots, x^{k}\right\}\right)$ with

$$
0 \in \operatorname{relint}(\bar{T}), R(\bar{T})=R(K) \text { and }\left\|x^{i}\right\|=R(K), 0 \leq i \leq k .
$$

Proof. cf. [BF], p. 9 and p. 54.

q.e.d.

With this lemma it is easy to find such $(d-1)$-dimensional planes for a simplex which produce the maximal $(d-1)$-circumradius with respect to projections or sections. 
Lemma 2. Let $T \in \mathcal{K}^{d}$ be a d-simplex, $\hat{F}$ a face of $T$ with maximal circumradius and $\hat{L} \in \mathcal{L}_{d-1}^{d}, \hat{x} \in \hat{L}^{\perp}$ with $\hat{x}+\hat{L}=\operatorname{aff}(\hat{F})$. Then

$$
\begin{aligned}
\text { i) } & R_{\sigma}^{d-1}(T)=R(T \cap(\hat{x}+\hat{L}))=R(\hat{F}), \\
\text { ii) } & R_{\pi}^{d-1}(T)=R(T \mid \hat{L})=R(\hat{F}) .
\end{aligned}
$$

Proof. Let $L_{d-1} \in \mathcal{L}_{d-1}^{d}$ with $R_{\pi}^{d-1}(T)=R\left(T \mid L_{d-1}\right)$ and let $T \mid L_{d-1}$ the convex hull of the points $x^{0}, \ldots, x^{d}$, where $x^{0}, \ldots, x^{d}$ denote the images of the vertices of $T$ under the projection onto $L_{d-1}$. Further let 0 be the center of the circumball of $T \mid L_{d-1}$ and $\bar{T} \subset T \mid L_{d-1}, \bar{T}=\operatorname{conv}\left(\left\{x^{0}, \ldots, x^{k}\right\}\right), 1 \leq k \leq d-1$, a $k$-simplex with the properties of lemma 1.

Now let $F$ be a face of $T$ containing such $k+1$ vertices which are mapped onto $x^{0}, \ldots, x^{k}$ with respect to the orthogonal projection onto $L_{d-1}$. We have

$$
R(\hat{F}) \geq R(F) \geq R\left(F \mid L_{d-1}\right) \geq R(\bar{T})=R_{\pi}^{d-1}(T) ;
$$

otherwise $R(\hat{F}) \leq R_{\sigma}^{d-1}(T) \leq R_{\pi}^{d-1}(T)$ and the assertion follows.

q.e.d.

On account of the lemma above we have $R(S) / R_{\pi}^{d-1}(S)=R(S) / R_{\sigma}^{d-1}(S)=d /\left(d^{2}-\right.$ $1)^{1 / 2}$ for a regular $d$-simplex $S$. That this is even an upper bound for every simplex is shown in the next lemma.

Lemma 3. Let $T \in \mathcal{K}^{d}$ a simplex. Then

$$
\begin{aligned}
\text { i) } \quad R(T) & \leq \frac{d}{\sqrt{d^{2}-1}} R_{\sigma}^{d-1}(T), \\
\text { ii) } \quad R(T) & \leq \frac{d}{\sqrt{d^{2}-1}} R_{\pi}^{d-1}(T),
\end{aligned}
$$

and equality holds if and only if $T$ is a regular $d$-simplex.

Proof. If $T$ is a regular $d$-simplex we have equality by lemma 2 . Hence on account of $R_{\sigma}^{d-1}(T) \leq R_{\pi}^{d-1}(T)$ it suffices to prove the lemma for the $(d-1)$-circumradius $R_{\sigma}^{d-1}(T)$.

Let 0 be the center of the circumball of $T$ and $\left\{x^{0}, \ldots, x^{k}\right\}$ a suitable subset of the vertices of $T$, such that $\bar{T}=\operatorname{conv}\left(\left\{x^{0}, \ldots, x^{k}\right\}\right)$ has the properties of lemma 1 . If $k<d$ then

$$
R(T)=R(\bar{T})=R_{\sigma}^{d-1}(T)<\frac{d}{\sqrt{d^{2}-1}} R_{\sigma}^{d-1}(T) .
$$

Hence we may assume that $T=\operatorname{conv}\left(\left\{x^{0}, \ldots, x^{d}\right\}\right)$ is a $d$-simplex with $0 \in \operatorname{int}(T)$ and $\left\|x^{i}\right\|=R(T), 0 \leq i \leq d$.

Let $\lambda$ be the maximal radius of a $d$-dimensional ball with center 0 , which is contained in $T$. This ball touches a face $F$ of $T$ in a point $\lambda a,\|a\|=1$. Let $F$ be given by $\operatorname{conv}\left(\left\{x^{1}, \ldots, x^{d}\right\}\right)$. Since $a$ is a normal vector of $\operatorname{aff}(F)$ we have

$$
\left\|x^{i}-\lambda a\right\|^{2}=R(T)^{2}-\lambda^{2}, \quad 1 \leq i \leq d .
$$

Hence $\lambda a$ is the center of the circumball of $F$ [BF, p. 54] and it follows

$$
R(T)^{2}-R_{\sigma}^{d-1}(T)^{2} \leq \lambda^{2} .
$$


For the inradius $r(T)$ of a simplex $T$ we have $r(T) \leq R(T) / d[\mathrm{~F}]$ and so by the choice of $\lambda$

$$
\lambda^{2} \leq \frac{R(T)^{2}}{d^{2}}
$$

Along with (2.2) this shows the inequality i). If we have equality in the relation i) then from (2.1), (2.2) and (2.3) follows that $T$ is a $d$-simplex with $r(T)=R(T) / d$. This is only possible if $T$ is regular $[\mathrm{F}]$. q.e.d.

Now we are able to prove the theorems.

Proof of Theorem 1. It obviously suffices to show the inequalities

$$
R_{\sigma}^{i}(K) \leq \frac{i}{\sqrt{i^{2}-1}} R_{\sigma}^{i-1}(K), \quad 1<i \leq d
$$

Since the circumradii are invariant with respect to translations we may assume that there is an $i$-dimensional linear subspace $L_{i} \in \mathcal{L}_{i}^{d}$ with $R_{\sigma}^{i}(K)=R\left(K \cap L_{i}\right)$ and 0 is center of the circumball of $K \cap L_{i}$. Moreover let $T \subset\left(K \cap L_{i}\right)$ a $k$-simplex with the properties of lemma 1 . Denoting by $R_{\sigma}^{i-1}\left(T ; L_{i}\right)$ the $(i-1)$-circumradius of $T$ with respect to the euclidean space $L_{i}$ we get from lemma 3

$$
R(T) \leq \frac{i}{\sqrt{i^{2}-1}} R_{\sigma}^{i-1}\left(T ; L_{i}\right)
$$

By the choice of $T$ we have $R(T)=R_{\sigma}^{i}(K)$ and since $R_{\sigma}^{i-1}(K) \geq R_{\sigma}^{i-1}\left(T ; L_{i}\right)$ the inequalities (1.1) are shown.

If an inequality of (1.1) is satisfied with equality for $i>j$ we must have equality in (2.4) and (2.5). By lemma 3 this means that $T$ is a regular $i$-simplex which satisfies the relation

$$
R(T)=R_{\sigma}^{i}(K)=\sqrt{\frac{i(j+1)}{j(i+1)}} R_{\sigma}^{j}(K) .
$$

Since $T$ is regular we have $R(T)=(i /(2 i+2))^{1 / 2} D(T)$ and by $(2.6)$ we see that $T$ has the diameter (edge length) $R_{\sigma}^{j}(K)((2 j+2) / j)^{1 / 2}$.

Now let $T$ be a regular $i$-simplex contained in $K$ with the given edge length. On account of (1.1) we get

$$
R(T)=\sqrt{\frac{i}{2 i+2}} D(T)=\sqrt{\frac{i(j+1)}{j(i+1)}} R_{\sigma}^{j}(K) \geq R_{\sigma}^{i}(K) .
$$

Clearly $R(T) \leq R_{\sigma}^{i}(K)$ and so we can replace ' $\leq$ ' by '=' in (2.7).

q.e.d.

Proof of Theorem 2. On account of lemma 3 the proof can be done in the same way as the proof of theorem 1 .

q.e.d. 


\section{Remarks}

(1) If we replace the first maximum condition by a minimum condition in the definition of the circumradii we get two other series of $i$-circumradii which now start with the half of the width of a convex body. If we further replace the circumradius by the inradius we totally get four series of circumradii and four series of inradii. Some of these functionals are studied in Computational Geometry $[\mathrm{GK}]$. For a survey of these generalized circumradii and inradii we refer to $[\mathrm{H}]$.

(2) Theorems involving inradius, circumradius, diameter and width have a long tradition in the geometry of convex bodies. In this context we refer to [BL], $[\mathrm{BF}],[\mathrm{E}],[\mathrm{DGK}]$.

\section{REFERENCES}

[BF] T. Bonnesen, W. Fenchel, Theorie der konvexen Körper, Springer, Berlin, 1934.

[BL] W. Blaschke, Kreis und Kugel, Veit; Second ed., W. de Gruyter, Berlin, Leipzig, 1916.

[DGK] L. Danzer, B. Grünbaum and V. Klee, Helly's theorem and its relatives. In Convexity (V. Klee, ed.), Amer. Math. Soc. Proc. Symp. Pure Math. 13 (1963), 101-180.

[E] H.G. Eggleston, Convexity, Cambridge Univ. Press, Cambridge, 1958, 1969.

[F] L. Fejes Tóth, Extremum properties of the regular polytopes, Acta. Math. Acad. Sci. Hungar. (1955), 143-146.

[GK] P. Gritzmann and V. Klee, Inner and outer $j$-radii of convex bodies in finite-dimensional normed spaces, to appear in Dis. and Comp. Geometry (1991).

[H] M. Henk, Ungleichungen für sukzessive Minima und verallgemeinerte In- und Umkugelradien konvexer Körper, Dissertation, Universitt Siegen (1991).

[J] H.W.E. Jung, Über die kleinste Kugel, die eine räumliche Figur einschließt, J. Reine Angew. Math. 123 (1901), 241-257.

Mathematisches Institut, Universität Siegen, Hölderlinstrasse 3, D-W-5900 Siegen Federal Republic of Germany. 